\title{
Oral Session 5: Motion Sickness and Pharmacology
}

\section{5-1 [\#3036]}

Validation of a Specific drug against G-Level transition induced spatial disorientation and orthostatic intolerance: The ESA SPIN-D study

A.P. Weerts ${ }^{\mathrm{a}}$, R. Vanspauwen ${ }^{\mathrm{a}}$, K.I. Buytaert ${ }^{\mathrm{a}}$, P.-F. Migeotte $^{b}$, X. Neyt ${ }^{b}$, P.H. Van de Heyning ${ }^{a}$ and F.L. Wuyts $^{\mathrm{a}}$

a Antwerp University Research centre for Equilibrium and Aerospace (AUREA), ENT Department, Antwerp, Belgium University Hospital, University of Antwerp, Antwerp, Belgium

${ }^{\mathrm{b}}$ Signal and Image Centre, Royal Military Academy, Brussels, Belgium

Spaceflight is known to induce vestibular and cardiovascular deconditioning. Consequently, astronauts suffer from orthostatic intolerance and spatial disorientation upon return to a gravitational environment. The aim of the current ESA SPIN-D study was to identify the most appropriate pharmacological countermeasure against the aforementioned symptoms.

This double blind placebo controlled study was conducted on 20 healthy, male volunteers. Tested medications were: baclofen $(10 \mathrm{mg})$, meclizine $(50 \mathrm{mg})$, dimenhydrinate $(40 \mathrm{mg})$ in combination with cinnarizine $(20 \mathrm{mg})$, promethazine $(25 \mathrm{mg}$ ) combined with dextroamphetamine $(5 \mathrm{mg})$. The effects of the drugs were investigated by a series of specific vestibular tests: elektronystagmography, cervical vestibular evoked myogenic potentials and unilateral centrifugation for canal, saccular and utricular function evaluation respectively. A head up tilt-test (HUT) was conducted in order to evaluate the cardiovascular response of the subjects during orthostatic stress.

Baclofen significantly increased the phase of the vestibulo-ocular reflex (VOR). This observation confirms the known inhibitory effect of baclofen on the ve- locity storage mechanism. Promethazine and dextroamphetamine caused a significant increase of the diastolic blood pressure (DBP) only during the supine part of the HUT, suggesting an influence of the combination on the activation of the vestibulo-sympathetic reflex when standing up. Based on the preliminary analysis of our data, we can conclude that the different medications seem to be acting on different, specific parts of the vestibular system.

\section{5-2 [\#3046]}

Pharmacokinetics of Scopolamine Intranasal gel Formulation (INSCOP) during antiorthostatic bedrest

L. Putcha ${ }^{\mathrm{a}}$, J.L. Boyd ${ }^{\mathrm{b}}$, B. Du ${ }^{\mathrm{c}}$, V. Daniels ${ }^{\mathrm{d}}$ and Z. Vaksman $^{\mathrm{e}}$

${ }^{a}$ NASA Johnson Space Center, Houston, TX, USA

${ }^{\mathrm{b}}$ Simulations Plus, Lancaster, CA, USA

${ }^{\mathrm{c}}$ Prairie View $A$ and $M$ University, Prairie View, TX, USA

${ }^{\mathrm{d}}$ Wyle Laboratories, Houston, TX, USA

${ }^{\text {e } U T ~ M e d i c a l ~ S c h o o l, ~ H o u s t o n, ~ T X, ~ U S A ~}$

Space Motion Sickness (SMS) is experienced during early flight days of space missions and on reduced gravity simulation flights which requires treatment with medications. Oral administration of scopolamine tablets is still a common practice to prevent SMS symptoms. Bioavailability of medications taken by mouth for SMS is often low and variable. Intranasal (IN) administration of medications has been reported to achieve higher and more reliable bioavailability than from an equivalent oral dose. In this FDA reviewed phase II clinical trial, we evaluated pharmacokinetics of an investigative new drug formulation, INSCOP during ambulatory $(\mathrm{AMB})$ and antiorthostatic bedrest 
(ABR), a ground-based microgravity analog. Twelve subjects including 6 males and 6 females received 0.2 and $0.4 \mathrm{mg}$ doses of INSCOP on separate days during $\mathrm{AMB}$ and $\mathrm{ABR}$ in a randomized, double blind cross over experimental design.

Blood samples were collected at regular time intervals for $24 \mathrm{~h}$ post dose and analyzed for free scopolamine concentrations by an LC-MS-MS method. Pharmacokinetic parameters were calculated using concentration versus time data and compared between AMB and ABR conditions. Results indicated that maximum concentration and relative bioavailability increased marginally during ABR compared to AMB; differences in PK parameters between AMB and ABR were greater with $0.2 \mathrm{mg}$ than with $0.4 \mathrm{mg}$ dose. Gender specific differences in PK parameters was observed both during $\mathrm{AMB}$ and $\mathrm{ABR}$ with differences higher in females between the two conditions than in males. A significant observation is that while gender differences in PK appear to exist, the differences in primary PK parameters between AMB and ABR after IN administration, unlike oral administration, are minimal and may not be clinically significant for both genders.

\section{5-3 [\#3003]}

\section{Postural prediction of motion sickness}

\section{T.A. Stoffregen}

Affordance Perception-Action Laboratory, University of Minnesota, 1900 University Ave. SE, Minneapolis, MN 55455,USA.E-mail: tas@umn.edu

Predicting motion sickness incidence is an inexact science. Existing methods rely on subjective data, such as motion history questionnaires, or on mathematical models that estimate sensory conflict. I offer a novel method that relies on a qualitatively different type of data. The subjective symptoms of visually induced motion sickness are preceded by changes in postural activity. This effect has been demonstrated in a wide range of settings including laboratory devices, fixed base flight simulators, virtual environments, head-mounted displays, and console video games. Effects occur during both standing and seated posture, and whether the observer has or does not have control of the visual motion stimuli. The robustness and generality of these effects suggest the possibility that motion sickness susceptibility might be predicted from data on postural activity. We [1] exposed standing subjects to oscillating op- tic flow in a moving room. We measured head movement prior to and during exposure. Using discriminant analysis, we developed equations that predicted motion sickness incidence (subjects' yes/no forced choice reports of whether they were motion sick) from specific parameters of head movement. These equations account for a higher percentage of the variance in subjective reports of motion sickness (up to 60\%) than is typically found with more traditional methods of prediction. Importantly, motion sickness incidence was predicted by body sway before subjects were exposed to the nauseogenic visual motion stimulus. This approach might be extended to predict motion sickness across different situations, including weightlessness.

\section{Reference}

[1] L.J. Smart, T.A. Stoffregen and B.G. Bardy, Human Factors 44 (2002), 451-465.

\section{5-4 [\#3056]}

\section{Cerebral blood flow decreases prior to nausea during off-vertical axis rotation}

\author{
J.M. Serrador ${ }^{\mathrm{a}, \mathrm{b}}$, M.J. Falvo ${ }^{\mathrm{a}}$, M. Blatt ${ }^{\mathrm{a}}$, J.J. Jasien ${ }^{\mathrm{a}}$ \\ and S.J. Wood ${ }^{\mathrm{C}}$ \\ ${ }^{a}$ War Related Illness and Injury Study Center, NJ VA \\ Health Care System, East Orange, NJ, USA \\ ${ }^{\mathrm{b}}$ Harvard Medical, Boston, MA, USA \\ ${ }^{\mathrm{c}}$ NASA Johnson Space Centre and Universities Space \\ Research Association, Houston, TX, USA
}

Nausea and motion sickness are important operational concerns for aviators and astronauts. Understanding underlying mechanisms associated with motion sickness may lead to new treatments. The goal of this work was to determine if changes in cerebral blood flow precede the development of nausea in motion sick susceptible subjects. Cerebral flow velocity in the middle cerebral artery (transcranial Doppler), blood pressure (Portapres) and end-tidal $\mathrm{CO} 2$ were measured while subjects experienced a $20^{\circ}$ off vertical axis rotation (OVAR) for $15 \mathrm{~min}$ at $0.1 \mathrm{~Hz}\left(36^{\circ} / \mathrm{sec}\right)$ followed by $15 \mathrm{~min}$ of $0.2 \mathrm{~Hz}(72 \% \mathrm{sec})$ rotation. Rotation was terminated when subjects reported persistent moderate nausea. Rotation while upright did not significantly change cerebral blood flow or blood pressure. Prior to nausea, subjects had significant increases in blood pressure $(+7.3 \pm 2.4 \mathrm{mmHg}, P<0.02)$ and cerebrovascu- 
lar resistance $(+17 \pm 2 \%, P<0.001)$ and decreases in cerebral flow velocity both in the second $(-5.2 \pm$ $2.1 \%)$ and last minute $(-4.9 \pm 1.8 \%)$ before termination $(P<0.001)$. In comparison, motion sick resistant subjects demonstrated no change in blood pressure, cerebrovascular resistance or cerebral flow velocity. These data indicate that cerebral hyoperfusion precedes the development of nausea. Further work is necessary to determine what role cerebral hypoperfusion plays in motion sickness. This work was supported by NASA grant NNJ04HI13G (Serrador) and NIH grant R21DC009900 (Serrador).

\section{5-5 [\#3047]}

\section{Are evolutionary hypotheses for motion sickness "Just So" stories?}

\section{C.M. Oman}

Man Vehicle Lab, Department of Aeronautics and Astronautics, MIT, Cambridge, MA, 02139-4307, USA. E-mail:coman@mit.edu

The survival advantage conferred in most vertebrates by powerful and easily conditionable nausea and vomiting reflexes as a third line of defense against ingested neuotoxins is relatively self-evident. In 1977 Triesman [1] proposed that ingestion of neurotoxins creates inconsistent vestibular, visual, and proprioceptive spatial referents, so linkages from sensory to emetic centers evolved as an additional defense mechanism. That certain motions also cause nausea and vomiting was considered an unfortunate epi-phenomenon. Triesman's hypothesis (aka the "Poison Theory") is certainly plausible, and is often cited as a scientific explanation for motion sickness. However after three decades it is fair to ask: how compelling is the evidence for it? Darwin [2,3] repeatedly cautioned that plausible evolutionary explanations are not always correct, since not every characteristic is adaptive. The notion that evolution shapes all phenotypic traits for survival advantage is referred to as "adaptationism" [4]. Kipling penned "Just So Stories" (e.g. "how the leopard got his spots") partly as parody. In evolutionary science, the term is now used to describe a plausible, but unverifiable /unfalsifiable evolutionary explanation for a specific phenotype. Certainly the generation of alternative plausible evolutionary hypotheses is an essential part of science - provided the hypotheses are testable. Thirty five years after Triesman proposed it, how com- pelling is the evidence for the "Poison Theory" [5]? Do ingested toxins typically cause vertigo? Vestibular sensory conflict neurons [6,7] exist whose primary role is arguably in body movement control, rather than vomiting. What about other plausible evolutionary explanations [8]? Alternatively, since vertebrate balance and emetic system neuroarchitectures are dictated by many potent phylogenic and ontogenic factors, could coupling between them in motion sickness simply be a maladaptive pleiotropic trait, recently manifested on an evolutionary timescale? Supported by the National Space Biomedical Research Institute through NASA NCC9-58.

\section{Reference}

[1] M. Triesman, Science 197 (1977), 493-496.

[2] C. Darwin, On the Origin of Species, John Murray, London, Ch. 6, 1857.

[3] C. Darwin, On the Descent of Man, John Murray, London, Ch. 6, 1871.

[4] S.J. Gould and R.C. Lewontin, Proc Royal Soc Lond (B) 205(1161) (1979), 581-598.

[5] K.E. Money and B.S. Cheung, Aviat Space Environ Med 54 (1983), 208-211.

[6] C.M. Oman, Can J Physiol Pharmacol 68 (1990), 294-303.

[7] K.E. Cullen, J.X. Brooks and S.G. Sadeghi, Ann NY Acad Sci 1164 (2009), 29-34.

[8] B. Bowins, Brain Res Bull 81 (2010), 7-11.

\section{5-6 [\#3060]}

\section{The evidence based etiology of Space Motion Sickness}

\section{W.E. Thornton}

Retired Astronaut, 17640 Pimlico Lane, Fair Oaks Ranch, Boerne, Texas, 78015,USA.E-mail: jwthornt@ msn.com

Space Motion Sickness (SMS) was reported on the second orbit of the second manned flight. Almost five decades later there is no satisfactory etiology [1]. Virtually all approaches to the problem to date are based on the concept of motions which produce conflicting responses from different sensory modalities $[1,2]$. Characteristic symptoms are triggered by such sensory conflicts through vestibular inputs to the chemo-receptor trigger zone Both symptoms and courses of motion sickness (MS) and SMS differ. SMS symptoms are consistent with demonstrated otolith organ function [3] and observed and recorded inflight effects [4]. The crux 
of vestibular effects of weightlessness is inherent in the graviceptor sensors of the otolith organs. Functionally they are weighted single axis pendulum accelerometers, spring loaded to a neutral position in the absence of displacing force. Thousands of these transducers are arranged geometrically to sense any orientation of the head to gravity. In weightlessness the variously oriented sensors all indicate a gravity vector normal to their mounting axis. This produces an intrinsic single modality sensory conflict which causes a paresis of the gastro-intestinal tract and produces the episodic vomiting and other symptoms characteristic of primary SMS [5]. Also, in weightlessness the otolithic sensors cannot produce their usual signals in response to angular head motion. This produces a bimodal sensory conflict with semi-circular canal signals, even with normal head motions and probably accounts for the characteristic of MS sometimes seen in SMS [2]. Thus weightlessness causes anomalous otolithic signals with both an intrinsic conflict and a bimodal motion dependent conflict. These produce differing symptoms apparently through different connections to the gastrointestinal system [4].

\section{Reference}

[1] G. Clement and M.F. Reschke, Neuroscience in Space 2008; Ch.3. Springer-Verlag, New York.

[2] J.W. Lackner and P. Di Zio, Space motion sickness, Exp Brain Res 175 (2006), 377-399.

[3] J.M. Goldberg and C. Fernandez, Physiology of peripheral neurons innervating otolith organs of the squirrel monkey. 1. Response to static tilts and to long duration centrifugal force, $J$ Physiol 39 (1976), 970-1008.

[4] W.E. Thornton, A Rationale for Space Motion Sickness ASEM 82(4) (2011), 467-468.

[5] W.E. Thornton, B.J. Linder, T.P. Moore and S.L. Pool, Gastrointestinal motility in space motion sickness, Aviat Space Environ Med 58(9 Suppl) (1987), A16-A21. 